\title{
Design and Finite Element Analysis of Electrical Plug Box Based on the Modular Mechanism
}

\author{
X.J. Zhang \\ Mechanical Engineering College of Northeast Dianli \\ University, Jilin \\ Jilin, China
}

\author{
X. J. Zhang \\ The Center of Workers Trained Jilin City Jilin \\ Jilin, China
}

\begin{abstract}
In this paper, the design of electrical plug box is discussed according the modular concept, its structure is analysed. The plug box is devisde, which is one module of a cabinet, using Solid Words. The cabinet is composed of bus board, modular power supply unit ( MPSU ), variable frequency speed regulation micro controller unit ( HVF-MCU ), data collection of analog input( DCAI ), analog output plug-in ( AO ), digital input plug-in ( DI ), digital output plug-in ( DO ) and human machine interface ( HMI ) and some standard parts assembled. The deformation of a plug-in box is analysised at different frequency and stress by ANSYS, pointing out the main factors of the deformation, improving the structure. The deformation is obviously reduced according to the results of analysis after the structure is improved.
\end{abstract}

Keywords-modularize; 3D design; finite element annlysis

\section{INTRODUCTION}

In response to product diversification and the current abbreviated trend of life cycles, modular product structure adopted by many product manufacturers that the final product (the system) is decomposed into modules (common parts) design and manufacturing division of labor. A large variety of new products and product systems constituted by different modules and flexible combination. The modular design of the mechanical structure is based on product function modules. Under the premise of the universal and the series, the product structure is divided into a number of combinations of modules. According to the product requirements, different modules quickly are combined into a complete product structure. The product requires structure, size and precision combination interface module interchangeability and substitutability because the module is often produced by a professional factory and circulates by the way the flow of goods.

The purpose of electronic equipment structural design is to make the system or equipment to meet the requirements of their electrical properties in a complex environment composed by machine, electromagnetism and thermal .At same time ,it also requires high reliability. Now days, Structural characteristics of electronic devices are putting plug box on top of the rack. In an electronic device, plug box is installed in the cabinet for mounting electronic components as a basic structure of the module, making the device as a whole on the basis of units. Structures with such features are suited to modular design pattern design [1]. Currently, the universal application of international multi-use electrical equipment size is using standard IEC297 family.

Plug box which is an important structural module can install and protect electronic devices. Generally, plug box is composed by the panel; base, electronic equipment, structures and traces oriented positioning support structure. At same time, it also needs light weight, suitable strength and stiffness. The design work and drawing of a Independent plug-in box is not great much, but an electronic system is often composed by dozens or even hundreds of plug-in boxes, and each plug-in box is different due to the different equipment, the overall design of the heavy workload and cumbersome. The quality of plug box design plays an essential role in a project [2]. Based on the structural features of plug-in box and Solidwords characteristics, the paper studied the automated design of plug-in box in Solidwords and the original design was improved on the basis of the finite element analysis.

In Solidwords, we can use Top-down design ideas to design plug box. Through basic correlation function of Solidwords, design intent can change the top-down pass until the bottom of the parts and drawings. It can improve modifiability of the product and reduce the workload greatly while ensuring the consistency of the design.

\section{THREE-DIMENSIONAL MODELING PLUG BOX}

Generally, the process of creating 3D model components is: creating a skeleton model - creating major parts based on the skeleton model and Layout files - assembling common parts based on skeleton model. Skel-model file is the first acquiescent element in the module assembly of Solidwords. Skel-model files can be created in the component while you can create a separate call later in the component. Plug-in box structure is not very complex. In order to facilitate changes, we use the method that is created in the establishment of the component inserting box Skel-model file.

After the skeleton model built, 3D models of the main parts that include panel, base and cushion strips were created. The entire processes are: creating part in the component model - copying a skeleton model to create solid model geometry released --Subrack. 1ay-- adds correspondence between three-dimensional model and Subtract. 1ay parameters.

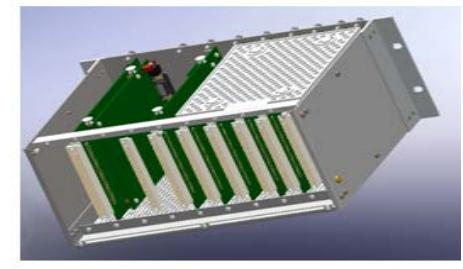

FIGURE I. 3D MODEL OF PLUG-IN BOX. 
Figure 1 is a three-dimensional model of plug-in box, which is mainly composed of the power plug MPSU, the main processor plug-HVF-MCU, analog acquisition plug DCAI, analog output plug-AO, digital input plug-DI and digital output plug-DO.

\section{FINITE ELEMENT MODAL ANALYSIS}

\section{A. The Pre-Treatment Process of Plug Box Three Modes by} ANSYS Analysis

The purpose of ANSYS modal analysis is to find the natural frequency of plug box, to avoid the use in the natural frequency and to prevent failure caused by vibrations [3].

The pre-treatment process of plug box three modes is completed by the following sections

(1) Setting analyze job name and title.

(2) Selecting the unit: the analysis to select 8-node tetrahedral solid structural units Quad 8 node 82.

(3) defining the real constants: taking unit thickness of $0.01 \mathrm{~m}$.

(4)defining material properties: the material parameters take Young's modulus (EX): 2.1e11; Poisson's ratio (PRXY): 0.3 .

(5) creating solid models:

First, a simplified plug box Solidwords model was established (Figure 2). Second, the feature model was imported used by the ANSYS import models. Then, the creation of a solid model was completed in ANSYS task. It is more simple and intuitive than the usual self with ANSYS to come.

(6) meshing:

We select "Areas" in the dialog box "Element Attributes" drop-down menu item and then select the small box before "SmartSize" where sets SmartSize value of 6 so that the system can set each side of the mesh size. After clicking the settings button in the line, the next step is to select the edge in the dialog box, point ok button and enter the number of nodes to be split in the pop-up dialog box. In accordance with the length of the edge, we enter 9, 7, 7, 5, 1, 1, click the "Mesh" button and select the model, OK. Mesh shown in Figure 3.

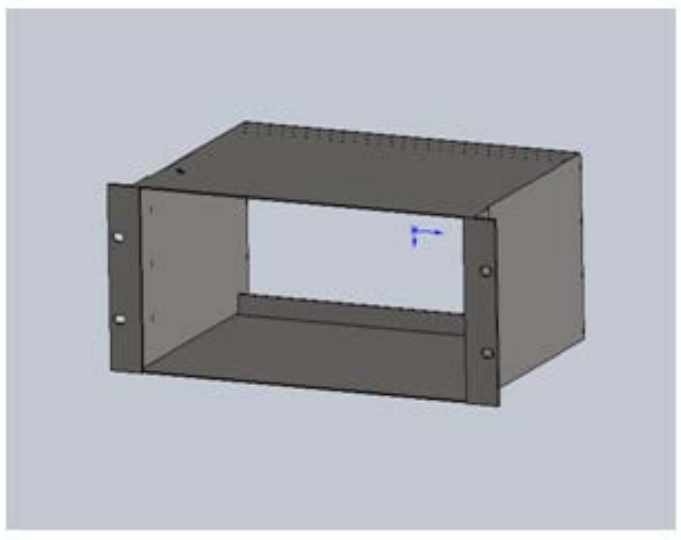

FIGURE II. THE SOLIDWORDS MODEL OF SIMPLIFIED BOX.

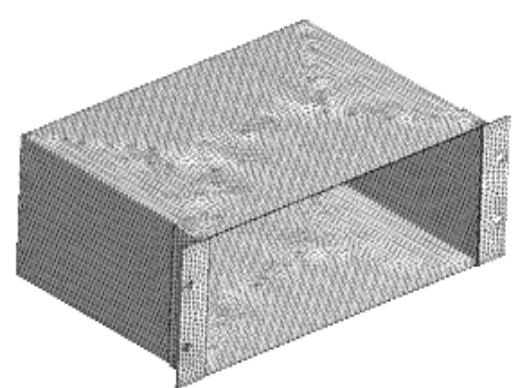

FIGURE III. THE MESHING OF SIMPLIFIED MODEL.

B. Modal Set, the Definition of Boundary Conditions and Solve Of Plug Box Three Modes Analysis

(1) Setting Mode

We select "Block Lanczos", enter 15 in No.of node to extract text box, make the Expand mode shaps No, enter 15 in No.of node to extract text box. Then we enter 0 in Start Freq text box, enter 574.84 in End Frequencey text box.

(2) defining the boundary conditions: both sides of the boundary selection.

(3) the solve of plug box three modes analysis

\section{Results of Plug Box Three Modes Analysis}

Three modes analysis results were Figure 4, Figure 5, Figure 6.

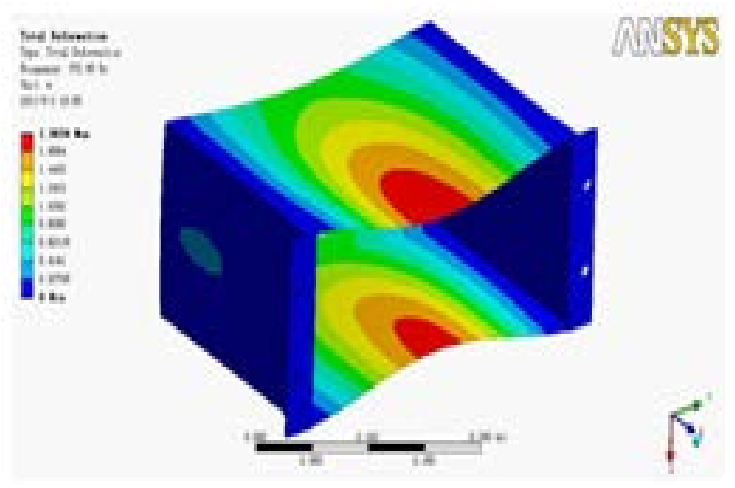

FIGURE IV. FIRST ORDER MODAL ANALYSIS OF BOX.

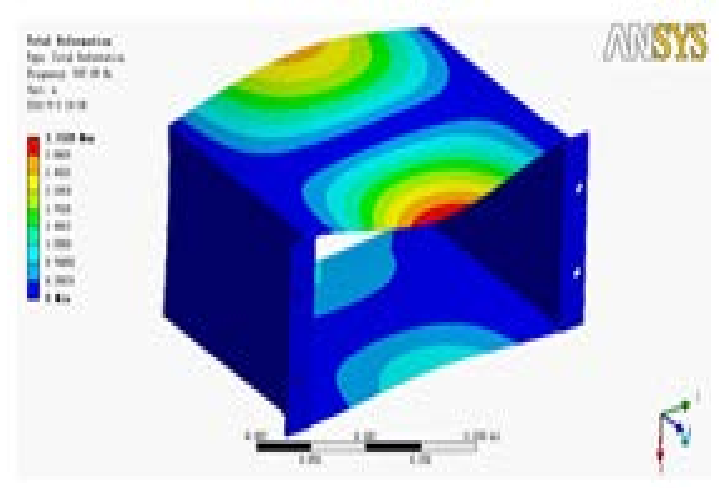

FIGURE V. SECOND ORDER MODAL ANALYSIS OF BOX. 

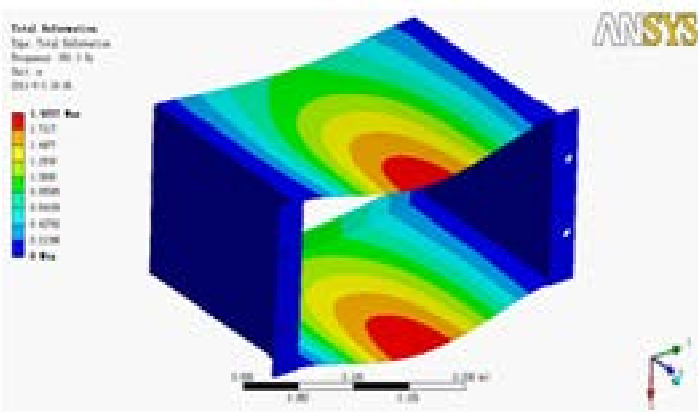

FIGURE VI. THIRD ORDER MODAL ANALYSIS OF BOX.

\section{Results of Plug-In Box Three Modes Analysis}

Through the analysis above, we can see the housing situation of the amplitude of the upper and lower surfaces at the frequency of $372.45 \mathrm{hz}, 391.3 \mathrm{hz}, 574.89 \mathrm{hz}$. The strongest amplitude is at the red part, while the deep blue is the weakest place. From the three modes of analysis above, it can be seen that the displacement of the plug box lower surface did not happen obviously at $372.45 \mathrm{hz}$. So did upper surface.

At $391.3 \mathrm{hz}$, the displacement of the plug box lower and the displacement is more significant than the first. At 574.89 $\mathrm{hz}$, the displacement of the plug box lower is more significant. From the above data, it can be seen that the normal circumstances should operate below frequency $372.45 \mathrm{hz}$. The plug-in box will have a greater amount of deformation while frequency is higher than $372.45 \mathrm{hz}$.

\section{FINITE ELEMENT ANALYSIS OF PLUG BOX STRESS}

The main purpose of stress analysis is to find the weakest position .According to the result; we can improve the design and the structural strength of the plug-in box. We can also optimize the structure [4].

The process of the plug box ANSYS stress analysis is same as analysis of its three modes. So no repeat is here. The results are shown in Figure 7.

The results of analysis show that the rear of the plug box is the weakest position for the entire structure. So the improvement of the structure will focus on improving the rear.

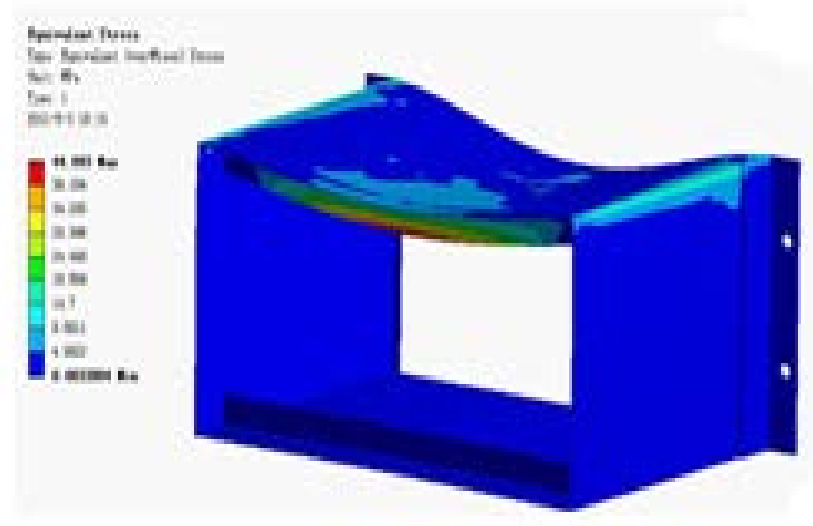

FIGURE VII. STRESS DISTRIBUTION.

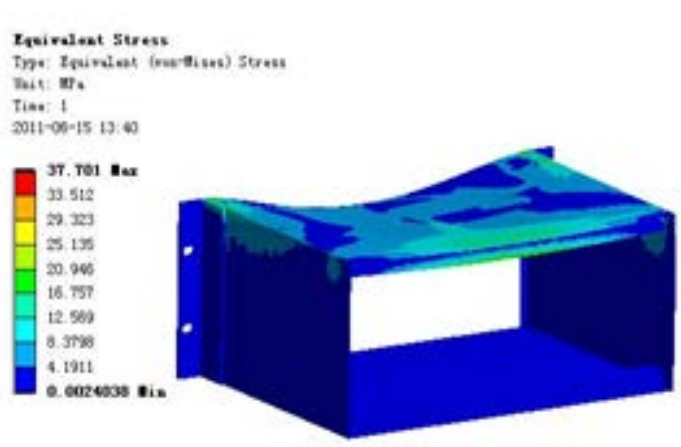

FIGURE VIII. IMPROVED STRESS PATTERN.

The purpose of the original design improvements is to improve the force situation of plug boxes or increase its rigidity. The principle of the improved design is: the improving process should not affect the normal use of plug-in box and ensure that its functionality is not affected. The smaller the changes to the original design are, the more excellent the improvements are. Through the analysis of the original design, we can find that the right angles to the rear baffle box plays the role of strengthening and improving the stiffness. In order to achieve little amount of change and same functions, the improved design uses a U-shaped slot instead of right angles slot in the original design. The results of Improvements stress analysis is in Figure 8.

Based on comparison of the analysis results in Figure 7 and Figure 8, it can be seen that subracks rear deformation significantly reduced after the right-angled baffle into channel-shaped baffle. So the improvement is feasible. It can be seen that the anti-destruction capability of subracks has a marked improvement. The program has been significantly optimized.

\section{CONCLUSIONS}

Through the analysis and research above, we can get the following conclusions:

(1) The use of 3D modeling solidworks can get available intuitive physical design drawings and CAD drawings can be generated by using solidworks. It provides a great convenience for the engineering whose drawing has no foundation or basis undoubtedly.

(2) We can use the correlation functions of the solidworks to get better modifications of the product and reduce the modified workload greatly while ensure the consistency of the design.

(3) It can be found that in order to avoid excessive deformation, plug box should be below $372.45 \mathrm{hz}$ frequency by ANSYS modal analysis.

(4) It can be seen that the weakest place of the entire structure is in the rear of the insert box by ANSYS stress analysis.

(5) The design modifications that use a modified rectangular-shaped baffle instead of channel-shaped baffle presented by this paper can improve the deformation capacity significantly improved by the analysis of ANSYS. 
In this study, we started from the modular design of the mechanism, used the insert box as the basic module, designed the basic module, pointed out the shortcomings of the original design and designed the modifications. Some parameters used in the design would not listed in the paper because of some technical reasons.

\section{REFERENCES}

[1] Zeng Wei, Tong Shizhong. Present Situation and Trends in Standardization of Mechanical Structures of Electronic Equipments.Electro-Mechanical Engineering. 2003,2:55-60

[2] JIN Xiaoli, WU Rong, ZHANG Aihua. Automatic Physical Design of Sub-rack by Pro/E. New Technology \& New Process, 2010,3: 20-24

[3] Guo Xuexun, Liang Rongliang, Chen Jian. Strength Analysis and Modal Analysis of Hydraulic Retarder Blade Based on ANSYS. Journal of Wuhan University of Technology (Transportation Science \& Engineering), 2010, 1(34):68-71.

[4] Wang Yongyan, Mao Huiqing, Zang Lin. Analysis of Stress and Model Pattern of Big Size Linear Vibration Screen. Coal Preparation Technology, 2010, 2:7-11. 